\title{
Environmental Enrichment Improves Response Strength, Threshold, Selectivity, and Latency of Auditory Cortex Neurons
}

Navzer D. Engineer, Cherie R. Percaccio, Pritesh K. Pandya, Raluca Moucha, Daniel L. Rathbun and Michael P. Kilgard

$J N$ 92:73-82, 2004. First published Mar 10, 2004; doi:10.1152/jn.00059.2004

You might find this additional information useful...

This article cites 56 articles, 13 of which you can access free at:

http://jn.physiology.org/cgi/content/full/92/1/73\#BIBL

This article has been cited by 2 other HighWire hosted articles:

Environmental Enrichment Increases Paired-Pulse Depression in Rat Auditory Cortex

C. R. Percaccio, N. D. Engineer, A. L. Pruette, P. K. Pandya, R. Moucha, D. L. Rathbun and M.

P. Kilgard

J Neurophysiol, November 1, 2005; 94 (5): 3590-3600.

[Abstract] [Full Text] [PDF]

Sound Case for Enrichment. Focus on "Environmental Enrichment Improves Response

Strength, Threshold, Selectivity, and Latency of Auditory Cortex Neurons"

H. R. Dinse

J Neurophysiol, July 1, 2004; 92 (1): 36-37.

[Full Text] [PDF]

Updated information and services including high-resolution figures, can be found at:

http://jn.physiology.org/cgi/content/full/92/1/73

Additional material and information about Journal of Neurophysiology can be found at: http://www.the-aps.org/publications/jn

This information is current as of December 2, 2005 .

Journal of Neurophysiology publishes original articles on the function of the nervous system. It is published 12 times a year (monthly) by the American Physiological Society, 9650 Rockville Pike, Bethesda MD 20814-3991. Copyright (C) 2005 by the American Physiological Society. ISSN: 0022-3077, ESSN: 1522-1598. Visit our website at http://www.the-aps.org/. 


\title{
Environmental Enrichment Improves Response Strength, Threshold, Selectivity, and Latency of Auditory Cortex Neurons
}

\author{
Navzer D. Engineer,* Cherie R. Percaccio,* Pritesh K. Pandya, Raluca Moucha, Daniel L. Rathbun, and \\ Michael P. Kilgard \\ Neuroscience Program, School of Behavioral and Brain Sciences, University of Texas at Dallas, Richardson, Texas 75083-0688
}

Submitted 16 January 2004; accepted in final form 8 March 2004

Engineer, Navzer D., Cherie R. Percaccio, Pritesh K. Pandya, Raluca Moucha, Daniel L. Rathbun, and Michael P. Kilgard. Environmental enrichment improves response strength, threshold, selectivity, and latency of auditory cortex neurons. J Neurophysiol 92: 73-82, 2004. First published March 10, 2004; 10.1152/jn.00059. 2004. Over the last $50 \mathrm{yr}$, environmental enrichment has been shown to generate more than a dozen changes in brain anatomy. The consequences of these physical changes on information processing have not been well studied. In this study, rats were housed in enriched or standard conditions either prior to or after reaching sexual maturity. Evoked potentials from awake rats and extracellular recordings from anesthetized rats were used to document responses of auditory cortex neurons. This report details several significant, new findings about the influence of housing conditions on the responses of rat auditory cortex neurons. First, enrichment dramatically increases the strength of auditory cortex responses. Tone-evoked potentials of enriched rats, for example, were more than twice the amplitude of rats raised in standard laboratory conditions. Second, cortical responses of both young and adult animals benefit from exposure to an enriched environment and are degraded by exposure to an impoverished environment. Third, housing condition resulted in rapid remodeling of cortical responses in $<2$ wk. Fourth, recordings made under anesthesia indicate that enrichment increases the number of neurons activated by any sound. This finding shows that the evoked potential plasticity documented in awake rats was not due to differences in behavioral state. Finally, enrichment made primary auditory cortex (A1) neurons more sensitive to quiet sounds, more selective for tone frequency, and altered their response latencies. These experiments provide the first evidence of physiologic changes in auditory cortex processing resulting from generalized environmental enrichment.

\section{N T R O D U C T I O N}

Environmental enrichment results in morphologic, molecular, and physiologic changes in sensory and motor cortices of young and adult animals (Diamond 2001; van Praag et al. 2000). Rats raised in enriched conditions exhibit increases in cortical thickness, gene expression, acetylcholinesterase levels, oligodendrocyte to neuron ratio, dendritic branching, and number of synapses per neuron compared with animals raised in standard conditions (Bennett et al. 1966; Diamond et al. 1966, 1972; Globus et al. 1973; Greenough et al. 1973; Hilbig et al. 2002; Rampon et al. 2000; Schapiro and Vukovich 1970; Sirevaag and Greenough 1987; Staiger et al. 2002; Volkmar and Greenough 1972).

* N. D. Engineer and C. R. Percaccio contributed equally to this work.

Address for reprint requests and other correspondence: M. P. Kilgard, Neuroscience Program, School of Behavioral and Brain Sciences, GR 41, Univ. of Texas at Dallas, Richardson, TX 75083-0688 (E-mail: kilgard@utdallas.edu).
Neurophysiologic responses can also be altered by experience. Experience-dependent remodeling of receptive fields and topographic organization of auditory cortex has also been observed in behaviorally trained animals (Ahissar and Ahissar 1994; Edeline 1999; Weinberger and Bakin 1998). Practice on frequency discrimination improved behavioral performance, narrowed receptive fields, and expanded the region of auditory cortex (A1) responding to the trained frequency (Recanzone et al. 1993). Cats raised in enriched conditions had sharper orientation tuning in primary visual cortex and were able to resolve higher spatial frequencies compared with cats raised in standard conditions (Beaulieu and Cynader 1990). Environmental enrichment narrowed receptive fields and sharpened the topographic organization of adult rat primary somatosensory cortex (Coq and Xerri 1998). The effects of more generalized enrichment have not been reported in auditory cortex.

In this study, our aims were to document the consequences of environmental enrichment on response properties of auditory cortex neurons and examine the time course of these changes. Evoked potentials from awake rats and extracellular recordings from anesthetized rats show significant changes in response strength, receptive field characteristics, and temporal response properties.

\section{METHODS}

\section{Environmental conditions}

Thirty-seven female Sprague-Dawley rats were used in this study. The standard housing condition consisted of one to two rats per cage (Fig. 1A). In the enriched environment, four to eight rats were housed together in a single large cage in a separate room from the main rat colony at the University of Texas at Dallas (Fig. $1 B$ ). All protocols and recording procedures conformed to the Ethical Treatment of Animals (National Institutes of Health) and were approved by the University Committee on Animal Research at the University of Texas at Dallas.

The enriched environment consisted of a large cage $(45 \mathrm{~L} \times 76$ $\mathrm{W} \times 90 \mathrm{H} \mathrm{cm}$ ) of four levels connected by ramps. Touch plates at the bottom of two ramps triggered different tones $(2,100$ or $4,000 \mathrm{~Hz})$ when the rat stepped on the plates. In addition, chains, wind chimes, or bells were hung across the entrance of each ramp so that a unique sound was elicited when rats passed from one level to the next. A motion detector emitted an electronic chime each time a rat crossed the infrared beam in front of the water source. An exercise wheel emitted a tone $(3,000 \mathrm{~Hz}$ Piezo Speaker) and activated a small green light emitting diode with each rotation. Each movement-triggered

\footnotetext{
The costs of publication of this article were defrayed in part by the payment of page charges. The article must therefore be hereby marked "advertisement" in accordance with 18 U.S.C. Section 1734 solely to indicate this fact.
} 
A STANDARD ENVIRONMENT

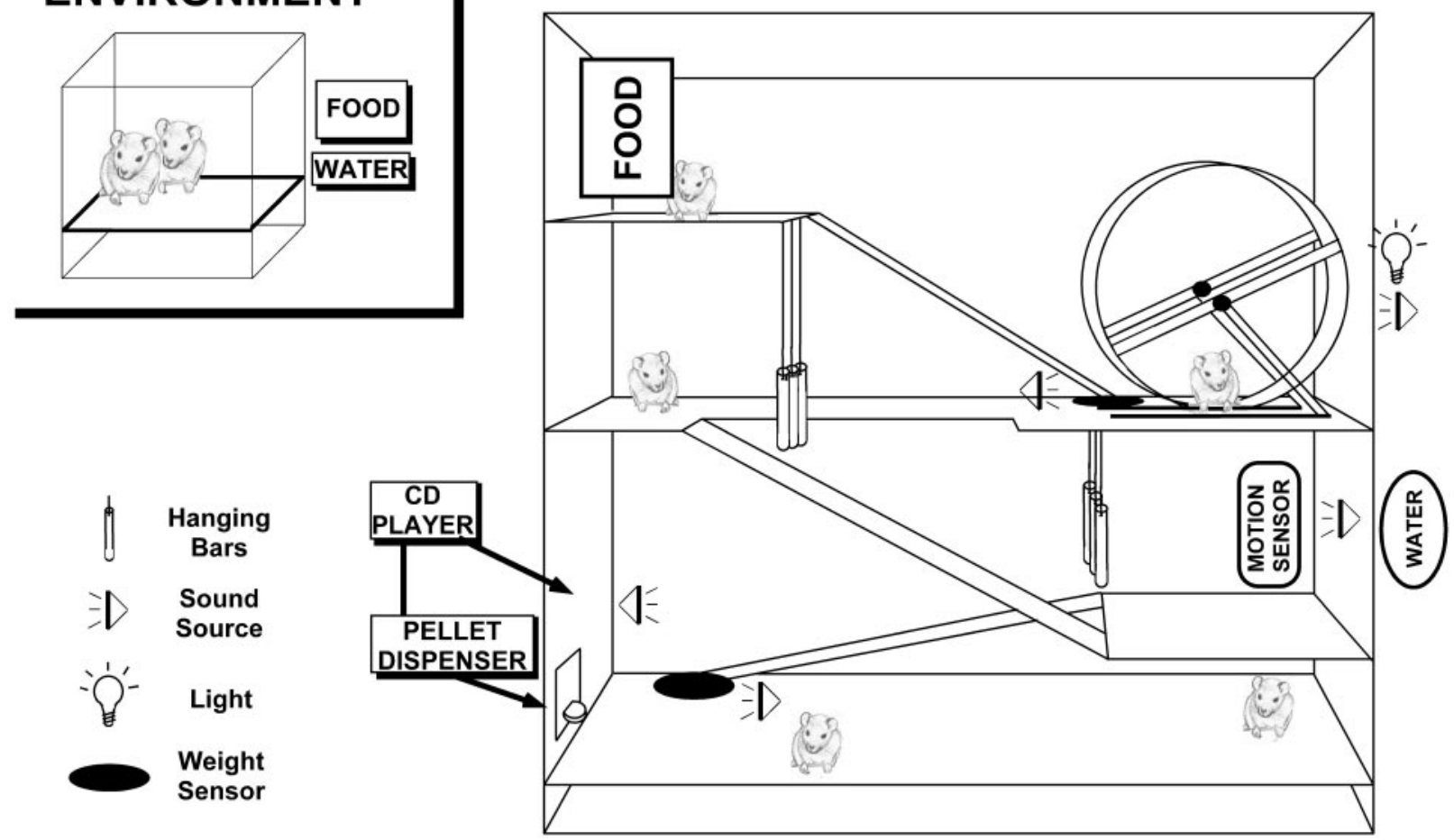

FIG. 1. Schematic of standard and enriched housing conditions. A: standard condition consisted of 1 or 2 rats housed in hanging cages within an animal colony room. $B$ : enriched condition consisted of 4-8 rats housed in a rich environment with devices that generated different sounds when rats crossed a motion detector path, stepped on weight sensors, or passed through hanging bars. In addition, each rotation of the running wheel triggered a brief tone and light flash, and a CD player played 74 sounds, including tones, noise bursts, musical sequences, and other complex sounds, in random order. Some of these sounds were associated with delivery of a sugar reward. While the sounds in the enriched environment were more diverse and behaviorally relevant, rats in both conditions heard approximately the same number of sounds each day.

sound had unique spectral and temporal characteristics that provided behaviorally meaningful information about the location and activity of other rats in the cage. The power spectrum of these sounds spanned the entire hearing range of the rat $(1-45 \mathrm{kHz})$. All sound intensities were $<75 \mathrm{~dB}$ SPL.

A CD player presented randomly selected sounds every $2-60 \mathrm{~s}$. These sounds included simple tones, amplitude-modulated and frequency-modulated tones, noise bursts, and other complex sounds (rat vocalizations, classical music, rustling leaves, etc.). Seven of the 74 sounds activated a pellet dispenser (Med Associates) that delivered a sugar pellet to encourage attention to the sounds. The rewarded tracks included modulated tones with different carrier frequencies and frequency-modulated sweeps. The sound sources added to the enriched environment were provided $24 \mathrm{~h} /$ day. After $1 \mathrm{mo}$, a vasectomized male rat was introduced into the enrichment cage to encourage more natural social interactions appropriate for these ages, since it is known that rats reach sexual maturation by $8-12$ wk of age.

The acoustic environment of the standard condition consisted of vocalizations from 20-30 other rats housed in the same room and sounds resulting from daily room traffic, feeding, and cleaning, which were also heard by the enriched group. However, sounds in the enriched condition were more diverse and provided more behaviorally relevant information than the sounds in the standard condition. Rats in both conditions were on a reverse 12-h light/dark cycle. As a result, both groups heard the sounds of room traffic while they were most active.

For both housing conditions, constant temperature and humidity were maintained. Food and water were provided ad libium for all rats. All the animals used in this study were housed with their mothers and littermates until weaning at $4 \mathrm{wk}$ of age.

\section{Experiment 1-extracellular recordings}

Thirty-day-old female Sprague-Dawley rats were randomly assigned to either the enriched environment $(n=8)$ or the standard condition $(n=8)$. The enriched rats were raised four per cage (in 2 sessions), while rats in the standard condition were raised two per cage. Acute microelectrode mapping was performed after $8 \mathrm{wk}$ in each environment (Fig. 2A). Although acute experiments using enriched and standard housed rats were interspersed, some experimenters were not blind to the identity (enriched or standard) of the animals because of the unkempt state of the rats' fur that typically identified the enriched rats. Hence, the possibility of unintentional bias cannot be completely excluded.

ACUTE SURGERY. Surgical anesthesia was induced with sodium pentobarbital $(50 \mathrm{mg} / \mathrm{kg}$, ip). A state of areflexia was maintained throughout the surgery and recording phases with supplemental doses of dilute pentobarbital ( $8 \mathrm{mg} / \mathrm{ml}$, ip). The interval between supplements varied depending on the anesthetic state of the animal but was typically every $1-1.5 \mathrm{~h}$. Anesthesia depth was evaluated by heart rate, breathing rate, toe-pinch responses, and corneal reflexes. These indicators were indistinguishable between the two groups. Circulatory function was monitored with EKG and pulse oximetry. Fluid balance was maintained with a 1:1 mixture of $5 \%$ dextrose and Ringer lactate $\left(\sim 0.5 \mathrm{ml} / \mathrm{h}\right.$ ). Body temperature was maintained at $37^{\circ} \mathrm{C}$. The trachea was cannulated to minimize breathing sounds and ensure adequate ventilation. Humidified air was delivered to the open end of the cannula. After the cisterna magnum was drained to minimize cerebral edema, the right auditory cortex was exposed, and the dura was resected. The cortex was maintained under a layer of viscous silicon 


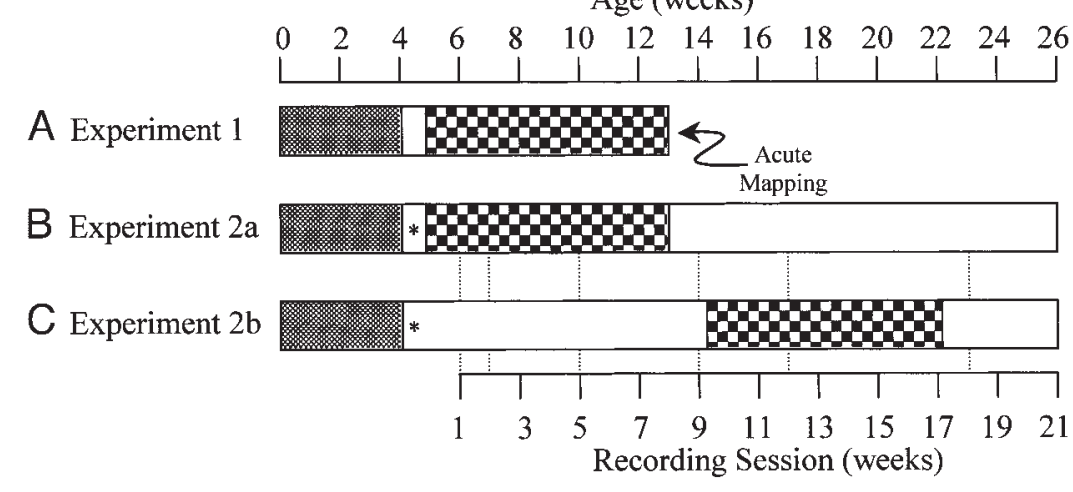

FIG. 2. Experimental timelines. A: experiment 1: extracellular recordings from rats housed in an enriched environment for 2 mo were compared with rats housed in the standard condition. $B$ and $C$ : experiment 2: evoked potentials from auditory cortex were recorded weekly for up to $5 \mathrm{mo}$. In experiment $2 a$ (early enriched), rats were housed in the enriched conditions for $8 \mathrm{wk}(4-12 \mathrm{wk}$ of age) and switched to standard conditions and housed singly until $26 \mathrm{wk}$ of age. In experiment $2 b$ (late enriched), rats were housed singly in the standard condition for 9 wk (4-13 wk of age), switched to the enriched condition for $8 \mathrm{wk}$, and then back to the standard condition until $26 \mathrm{wk}$ of age. *Time of chronic electrode implantation.
Housed $w /$ mother and littermates

\section{Standard} Environment

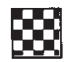

Enriched Environmen oil to prevent desiccation during the $24-30 \mathrm{~h}$ experiment, and a detailed map of auditory cortex was generated from 50-100 microelectrode penetrations. The sampling density and depth of recordings made in enriched and standard housed rats were indistinguishable. Parylene coated tungsten microelectrodes (FHC) were lowered 550 $\mu \mathrm{m}$ below the pial surface (layer $4 / 5$ ) of the right auditory cortex. Spikes from a small cluster of neurons were collected at each penetration site. Penetration locations were referenced using cortical vasculature as landmarks.

STIMULUS PRESENTATION AND DATA ANALYSIS. Auditory stimuli were delivered from the left side of the rat via a calibrated speaker in a shielded, double-walled sound attenuating chamber and were generated using Brainware (Tucker-Davis Technologies). Auditory frequency tuning curves were determined at each site by presenting 81 logarithmically spaced frequencies from 1 to $32 \mathrm{kHz}$ at 16 intensities from 0 to $75 \mathrm{~dB}$ (1,296 total stimuli). The tones were randomly interleaved and separated by $500 \mathrm{~ms}$. In addition, responses to $70-\mathrm{dB}$ noise burst trains ( 4 rates: $5,10,15$, and $20 \mathrm{~Hz} \times 12$ repetitions) were also recorded at each penetration site. A population poststimulus time histogram (PSTH) was created by summing spikes resulting from the repeated presentation of a single noise burst at each site. All stimuli were $25 \mathrm{~ms}$ long with a 3 -ms rise and fall time. Action potentials were recorded simultaneously from two Parylene-coated tungsten microelectrodes $(2 \mathrm{M} \Omega)$. The neural signals were filtered $(0.3-15 \mathrm{kHz})$ and amplified (10,000 times). Action potential waveforms were recorded whenever a set threshold was exceeded.

Tuning curve parameters were defined by an experienced blind observer using custom software that randomized the order of data from each recording site across both groups. Best frequency (BF), bandwidth measures (BW), response threshold, spontaneous rate, and latency measurements for each penetration were recorded (Fig. 3). The CF is the frequency that evokes a reliable response at the lowest intensity (response threshold). Frequency bandwidth is the range of frequencies that each site responds to at $10,20,30$, and $40 \mathrm{~dB}$ above threshold. First spike latency is the time from stimulus onset to the earliest reliable neural response. The end of response was defined as the time after tone onset when the PSTH created by summing the responses to all of the tones within each site's tuning curve returned to baseline. The borders of A1 were defined based on continuous topography of CF and short response latency. Sites with high thresholds, long latencies, broad tuning, and discontinuities in CF topography were considered non-A1 (Doron et al. 2002; Kilgard et al. 2001). Criteria for identifying non-A1 sites were subjective and were applied blindly by well-trained observers.

Cortical maps were reconstructed using the Voronoi tessellation procedure. The percent of A1 responding is the sum of the areas of all of the Voronoi tessellations that responded to the particular frequency and intensity combination divided by the total area of A1 (Kilgard and
Merzenich 1998). The tessellation procedure generates polygons from a set of nonuniformly spaced points so that every point in the polygon was nearer to the sampled point than to any other. This allowed area information to be estimated from a number of discretely sampled recording sites by assigning each point on the cortical surface the qualities of the closest sampled point. The area measures generate reliable estimates of the percent of primary auditory cortex that is responsive to a given frequency-intensity combination.

\section{Experiment II-auditory-evoked potential}

EXPERIMENTAL TIME COURSE. Evoked potentials were recorded each week from an electrode implanted over left auditory cortex to determine the time course of environmental plasticity. Twenty-one female Sprague-Dawley rats were randomly assigned to one of two groups. Rats in experiment $2 a(n=12)$ were housed in the enriched environment for $8 \mathrm{wk}(4-12 \mathrm{wk}$ of age) and then moved to the standard condition and housed singly until $26 \mathrm{wk}$ of age (Fig. 2B). Rats in experiment $2 b(n=9)$ were housed singly in the standard condition for $9 \mathrm{wk}$ (4-13 wk of age), moved to the enriched environment for $8 \mathrm{wk}$, and then back to the standard condition until $26 \mathrm{wk}$ of age (Fig. 2C).

CHRONIC IMPLANTATION. Rats were chronically implanted at 28 days of age with a ball electrode over AI and a ground screw over the cerebellum. Surgical anesthesia was induced with sodium pentobarbital $(50 \mathrm{mg} / \mathrm{kg}$, ip). A state of areflexia was maintained throughout the surgery, and supplemental doses of dilute pentobarbital were administered subcutaneously if needed ( $8 \mathrm{mg} / \mathrm{ml}$, ip). Anesthesia level was monitored by response to toe pinch. Atropine and dexamethasone were administered subcutaneously to minimize lung secretions and brain edema during the procedure and recovery. Animals received antibiotic injections (ceftriaxone) before and after surgery. Body temperature was measured by a rectal probe and maintained at $37^{\circ} \mathrm{C}$. Four to five structural screws were used to anchor the implant on the skull. The 4-pin connector was held in place with dental acrylic. Although some implants remained firmly in place for 5 mo, many implants were lost due to skull growth following implantation. Implanted rats were housed singly when not in the enriched environment to eliminate the tendency of rats in small cages to damage implants by excessive grooming.

STIMULUS PRESENTATION AND DATA ANALYSIS. Middle latencyevoked potential data were collected once each week for $21 \mathrm{wk}$ from each rat while placed in a sound-attenuated booth. Recordings were made during the dark cycle in both housing conditions to encourage the rats to be as alert as possible, but the rats did spend some time sleeping. EEG recordings indicate rats were in slow wave sleep no more than $25 \%$ of the time. Overall, no differences in activity levels, 
A

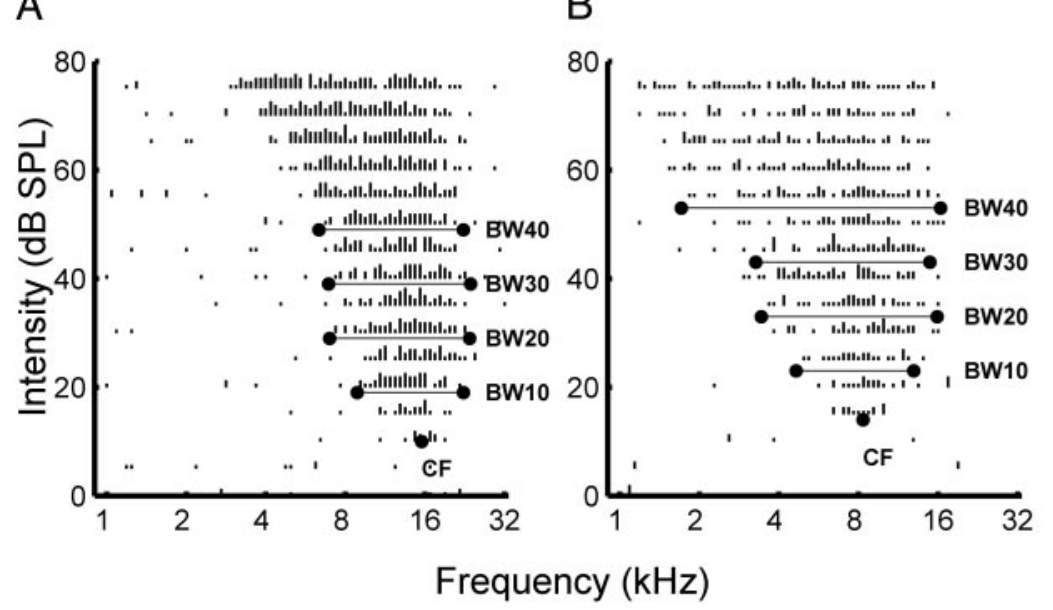

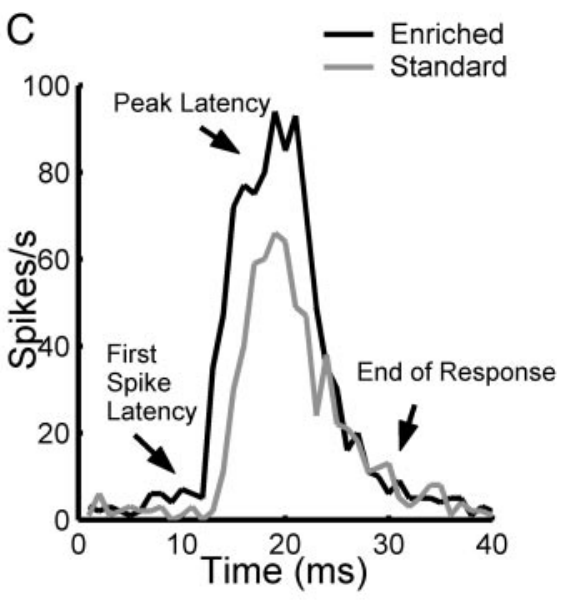

FIG. 3. Tone responses from an enriched rat $(A)$ show more spikes per tone, narrower tuning, and lower thresholds compared with tone responses from a standard rat $(B)$. $C$ : poststimulus time histograms (PSTHs) for these 2 sites show greater response strength after enrichment. Binwidth is $1 \mathrm{~ms}$.

exploration, time spent sleeping, or arousal levels were noted between enriched and standard rats during recording sessions. Tones and noises were presented from a speaker centered above the cage and randomly interleaved every $10 \mathrm{~s}$. Stimuli included a $25-\mathrm{ms}, 9-\mathrm{kHz}-$ tone, 100-ms loud (70 dB SPL) and quiet (50 dB SPL) noise bursts with $3-\mathrm{ms}$ rise and fall times. These sounds were not identical to any of the sounds heard in the enrichment cage. Since there was no specific overrepresentation of the exposed frequencies of the enriched A1 map when assayed using microelectrode mapping, we expected similar results in the evoked potential data (i.e., a nonspecific increase in cortical response strength). Therefore $9 \mathrm{kHz}$ was selected for the evoked potential study because it was not presented as part of the enriched environment. Thus rats did not hear the $9-\mathrm{kHz}$ tone or noise burst outside of the evoked potential recording sessions. Signals were amplified (10,000 times) and displayed on an oscilloscope for monitoring (filtered $0.1-800 \mathrm{~Hz}$ ). Data acquisition computers collected 250 traces of each tone and 125 traces of each noise. Trials with excessive motion artifacts $(>0.1 \mathrm{mV})$ were discarded prior to analysis of the mean evoked potential.

The first two negative peaks in the evoked response are referred to as the $\mathrm{N} 1$ and $\mathrm{N} 2(\sim 40$ and $140 \mathrm{~ms}$, respectively; Fig. $5 A)$. The first positive peak is referred to as $\mathrm{P} 1(\sim 75 \mathrm{~ms})$. The $\mathrm{N} 1$ potential in response to the tone was composed of two distinct subpeaks, which we refer to as N1a and N1b ( $\sim 25$ and 45 ms, respectively; Fig. $6 B)$.

The sum of the absolute values of the evoked potential (30-100 ms after sound onset) was used to quantify the size of the auditory-evoked response. To eliminate variability in evoked potential amplitude due to individual differences in electrode position and recording characteristics, the size of the evoked response for each week was divided by the size on week 1. Logarithm base two of this ratio was used so that -1 and +1 indicates twofold decreases and increases, respectively. Statistical significance was determined throughout this report using Student's $t$-test.

\section{RE S U L T S}

Neurophysiologic responses were recorded from rats housed either in standard laboratory conditions or in an enriched environment (see METHODS and Figs. 1 and 2). Action potentials were recorded from small groups of A1 neurons at $>800$ sites in 16 rats. Twenty-one rats were implanted with EEG electrodes, and evoked responses were recorded from auditory cortex each week for up to 5 mo. By combining these two techniques, we were able to document the effects of environmental enrichment in fine spatial detail and over time.

\section{Extracellular recordings (experiment 1)}

Although rats in the enriched environment were exposed to a variety of broadband and narrowband stimuli, environmental plasticity was quantified by recording A1 responses to simple tones and white noise bursts. Comparisons between responses from animals housed in enriched ( $n=8$ rats; 462 sites) and standard ( $n=8$ rats; 358 sites) conditions indicated that environment substantially altered response strength, receptive field size, intensity threshold, spontaneous rate, and response latency. Enrichment did not significantly alter the A1 map of tone frequency. In both groups, best frequency was highly correlated with anterior-posterior position $\left(r^{2}=0.83 \pm 0.02\right.$ and $0.82 \pm 0.03$ for enriched and standard, respectively). The average change in best frequency as a function of anteriorposterior distance was not significantly different between the two groups $(-0.31 \pm 0.01$ and $-0.27 \pm 0.02$ octaves $/ \mathrm{mm}$ for enriched and standard, respectively). The total size of A1 was also unaffected by enrichment $[1.03 \pm 0.17$ (enriched) and $0.80 \pm 0.15 \mathrm{~mm}^{2}$ (standard), $\left.P>0.05\right]$. No overrepresentation of any frequency-intensity combination (including the 2.1-, 3-, or $4-\mathrm{kHz}$ frequencies used in the enriched condition) was observed (see METHODS).

The number of action potentials in response to tones or noises increased by one-third in enriched rats compared with rats housed in the standard condition (Table 1). A1 neurons in enriched rats were nearly $3 \mathrm{~dB}$ more sensitive to tones and were more selective for tone frequency than neurons in the standard group (Table 1; Fig. 3). Bandwidth at 10-40 dB above threshold was narrower for enriched rats, but only reached statistical significance at $40 \mathrm{~dB}$ above threshold. End of response latency was later as a result of the greater number of spikes evoked by each sound (Table 1; Fig. 4). The average signal to noise ratio increased by $11 \%$ due to the greater rise in driven rate over spontaneous rate. Spontaneous activity in enriched rats was $21 \%$ higher. Each of these changes were observed in all regions of the frequency map.

\section{Auditory-evoked potentials (experiments $2 a$ and $2 b$ )}

To document the effect of behavioral state and determine the time course of environmental plasticity, auditory-evoked po- 
TABLE 1. Response properties of primary auditory cortex neurons recorded from rats housed in enriched and standard conditions

\begin{tabular}{|c|c|c|c|}
\hline Response Parameter & $\begin{array}{c}\text { Enriched } \\
(N=462 \text { A1 sites })\end{array}$ & $\begin{array}{c}\text { Standard } \\
(N=358 \text { A1 sites })\end{array}$ & $P$ Value \\
\hline Response strength (spikes/tone) & $1.61 \pm 0.04$ & $1.24 \pm 0.06$ & $<0.00001$ \\
\hline Response strength (spikes/noise) & $1.44 \pm 0.04$ & $1.04 \pm 0.06$ & $<0.00001$ \\
\hline Neural threshold (dB) & $17.19 \pm 0.47$ & $19.88 \pm 0.61$ & $<0.001$ \\
\hline RF bandwidth (BW40) & $2.01 \pm 0.04$ & $2.16 \pm 0.05$ & $<0.05$ \\
\hline Peak latency (ms) & $19.18 \pm 0.18$ & $18.32 \pm 0.25$ & $<0.01$ \\
\hline End of peak latency (ms) & $36.09 \pm 0.39$ & $34.41 \pm 0.40$ & $<0.01$ \\
\hline Spontaneous (spikes/20 ms) & $0.114 \pm 0.004$ & $0.094 \pm 0.006$ & $<0.01$ \\
\hline
\end{tabular}

Values are mean $\pm \mathrm{SE}$. Student's $t$-tests were used to determine statistical significance.

tentials were recorded each week for 21 wk from rats implanted with chronic epidural electrodes (Fig. 2). Following implantation, rats recovered for $1 \mathrm{wk}$, after which they were randomly assigned to either the enriched or standard housing condition.

NOISE-EVOKED RESPONSES. A 70-dB noise burst resulted in negativities 40 and $140 \mathrm{~ms}$ after noise onset (N1 and N2) and a positivity $75 \mathrm{~ms}$ after onset (P1; Fig. $5 A$ ). The shapes of the grand mean averaged evoked potentials from each group were similar during the first few weeks of differential housing (Fig. 5, $A$ and $B$ ). After 5 wk of enrichment, the average evoked potential amplitude from the enriched group was twice the size of the standard housing group (Fig. 5C). When the enriched group was transferred to the standard environment at $13 \mathrm{wk}$ of age, the amplitude of the auditory evoked potential was reduced by $60 \%$ within 1 wk of moving (Fig. 5, $C$ and $D$ ). When the standard condition group was transferred to the enriched environment, the response amplitude doubled within $1 \mathrm{wk}$ (Fig. 5, $D$ and $E$ ). The noise-evoked responses of the early and late enriched

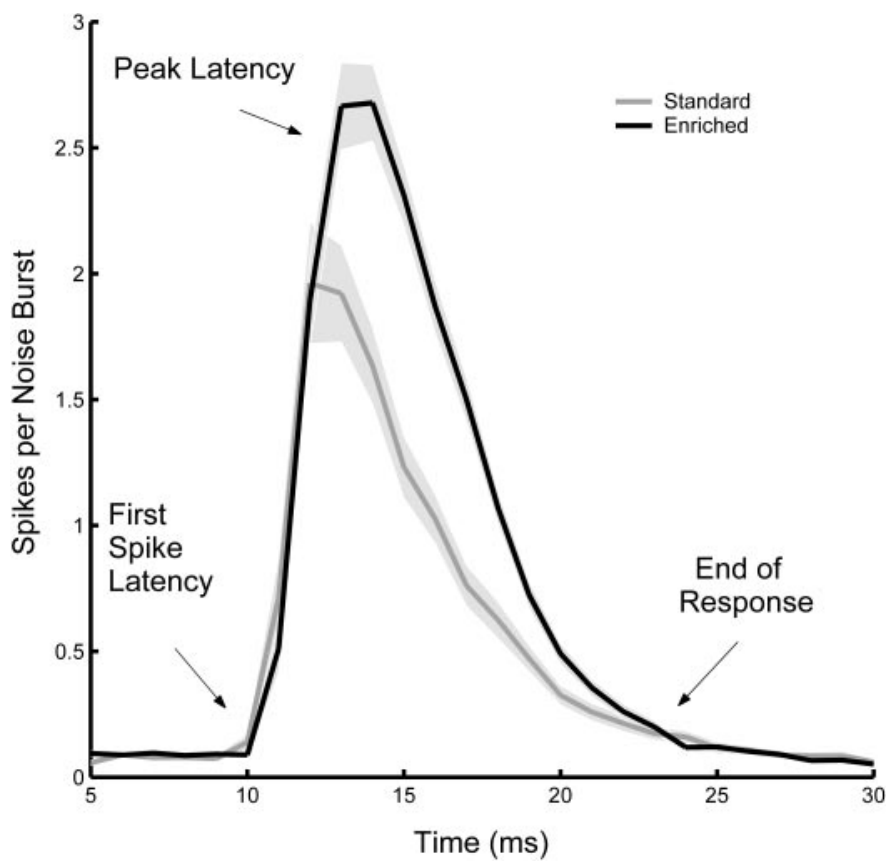

FIG. 4. Mean noise burst PSTHs for $N($ enriched $)=462$ and $N($ standard $)=$ 358. Enriched rats exhibit a higher firing rate, late peak, and end of response latency. Gray shaded regions represent the SE for each group. Binwidth is 1 ms. groups were indistinguishable when both were housed in the standard environment at weeks 9 and 18 (Fig. 5, $D$ and $F$ ).

Since responses of animals in the standard condition decreased over the first several weeks while responses of enriched animals were unaltered, it appears that enrichment may protect cortical responses from decline rather than strengthening them (Fig. 5, $A-C$ ). Alternatively, it may not be possible to increase the response to noise bursts since nearly all neurons in A1 already respond to these broadband sounds. To avoid this potential ceiling effect, we also quantified the cortical response to a $9-\mathrm{kHz}$ tone $(70 \mathrm{~dB})$, which activates approximately onehalf as many A1 neurons (Kilgard and Merzenich 1999).

TONE-EVOKED RESPONSES. As expected, responses to tones were significantly smaller than noise-evoked responses. Differences in tone-evoked response amplitude were evident after as little as 2 wk of differential housing (Fig. 6, $A$ and $B$ ). The tone-evoked potentials from the standard group decreased by nearly twofold over the first several weeks of the experiment (Fig. 6, $A$ and $C$ ). In contrast, the tone-evoked potential from the enriched group increased to nearly twice its initial peakto-peak amplitude. Both P1 and N1 amplitudes were significantly larger for the early enriched group compared with the group housed in the standard environment (P1: $0.07 \pm 0.01$ vs. $0.03 \pm 0.01 \mathrm{mV}, P<0.05 ; \mathrm{N} 1:-0.05 \pm 0.01$ vs. $-0.03 \pm$ $0.01 \mathrm{mV}, P<0.05)$. Similar changes were also observed when the responses of individual animals were compared before and after enrichment. This paired analysis revealed a significant increase in amplitude for both $\mathrm{P} 1(0.03 \pm 0.01 \mathrm{mV}, P<0.005)$ and N1 $(-0.02 \pm 0.01 \mathrm{mV}, P<0.05)$. The strengthening of the tone-evoked response occurred in both young and adult rats (Fig. 6E).

The N1 component of the tone-evoked response exhibited two distinct subpeaks not seen in responses to noise (Fig. 6B). Most of the difference in N1 amplitude due to differential housing resulted from changes in the N1b (Fig. 6C). The N1b in enriched rats was nearly twice as large after 5 wk compared with week 1, while the $\mathrm{N} 1 \mathrm{~b}$ in standard housed rats was completely absent from weeks 2-9 (Fig. 6, B-D). The N1b developed within $1 \mathrm{wk}$ of moving standard housed animals to the enriched environment (Fig. 6, $D$ and $E$ ). While the response amplitude from both groups was reduced when they were moved to the standard environment, the N1b was still evident many weeks later (Fig. 6F). These results indicate that some effects of enrichment may endure beyond the period of enrichment. However, most aspects of the auditory-evoked response reflect environmental conditions during the prior week.

As a result of the observed increase in N1b amplitude, the $\mathrm{N} 1$ peak latency was found to increase with enrichment 


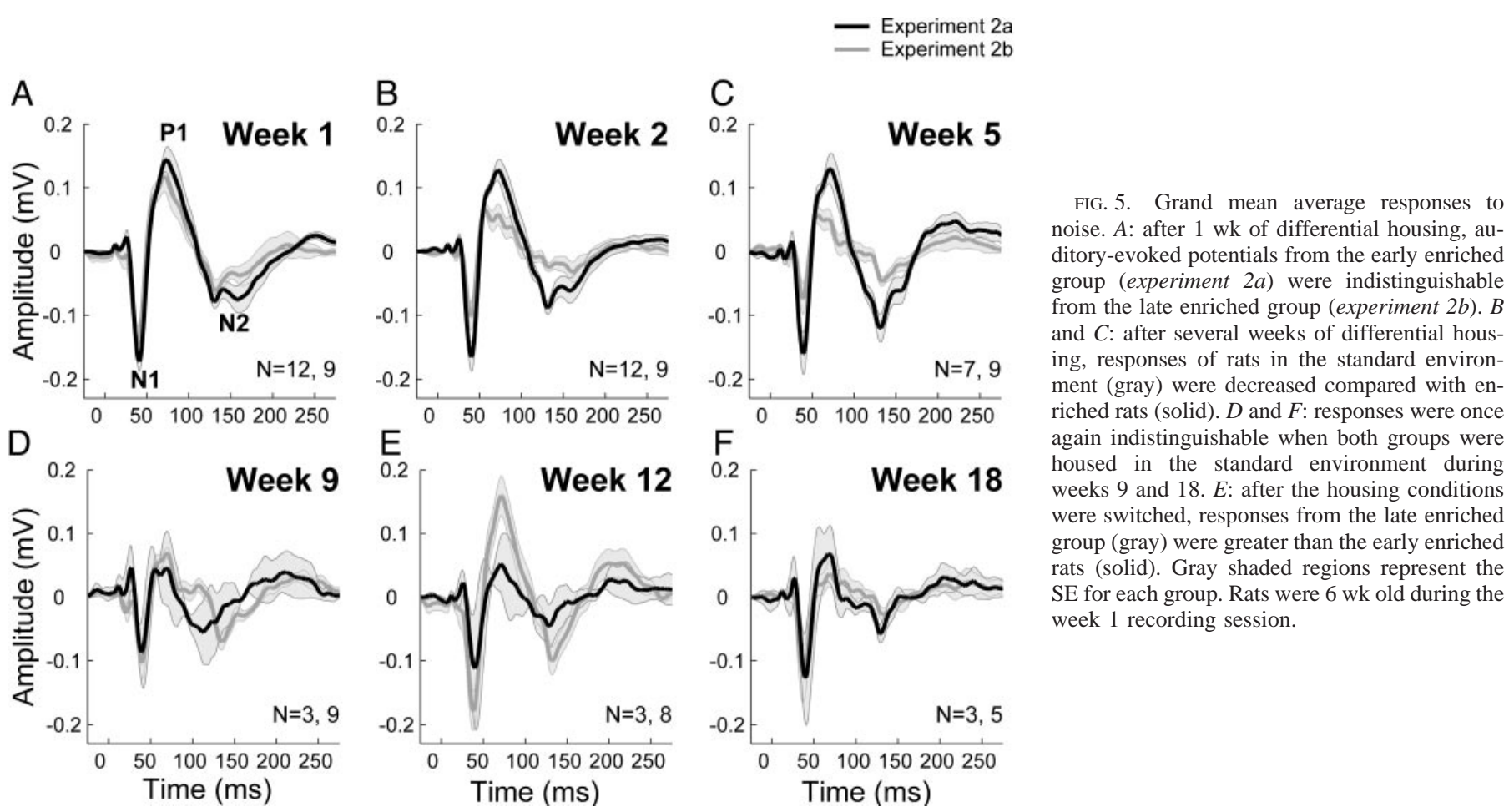

( $33.55 \pm 2.58$ vs. $23.44 \pm 0.83 \mathrm{~ms}, P<0.01)$. Similar changes were also observed when the latencies from individual animals were compared before and after enrichment $(7.70 \pm 3.30 \mathrm{~ms}$, $P<0.05)$. These results are consistent with data from the acute experiment in which strengthened responses exhibited later peak latencies. Interestingly, the latency of the P1 decreased in the enriched group compared with the standard housed group $(69.73 \pm 1.88$ vs. $88.22 \pm 8.93 \mathrm{~ms}, P<0.01)$. Although the neural generator of the $\mathrm{P} 1$ is not clear, it is possible that the decrease in P1 latency due to enrichment results from earlier activation of nonprimary cortical fields.

TIME-COURSE OF AUDITORY-EVOKED PLASTICITY. Normalizing each individual's responses to their week 1 response eliminates variability due to electrode placement and facilitates direct comparison of tone and noise response plasticity. Figure 7 shows the relative changes in tone- and noise-evoked responses over time for each experimental group. A plasticity index value
- Experiment 2a Experiment 2b
A

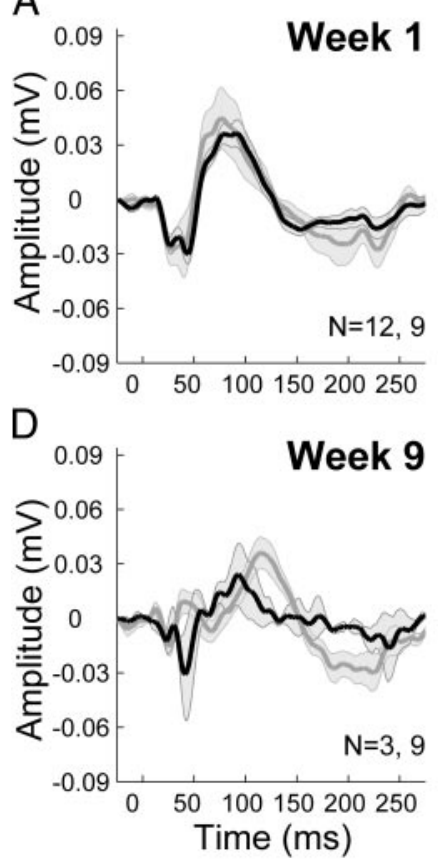

$B$
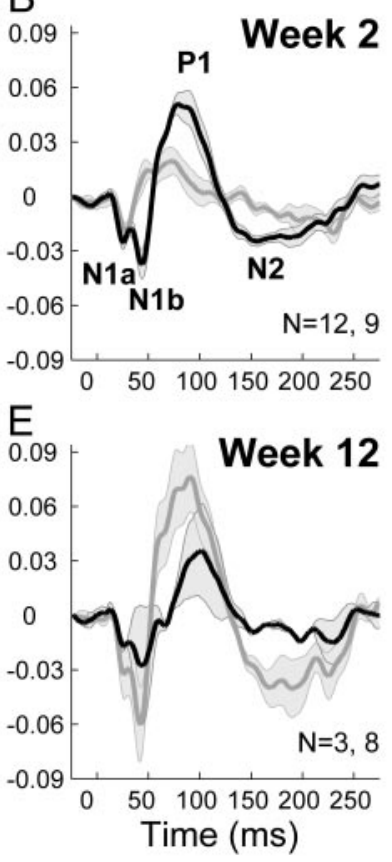

C

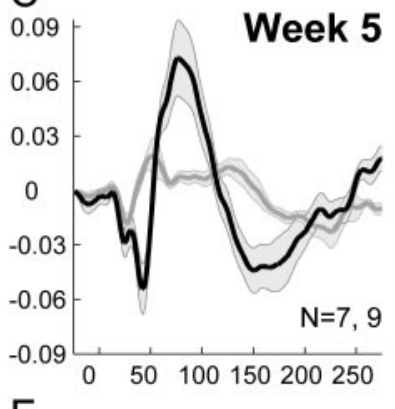

$F$

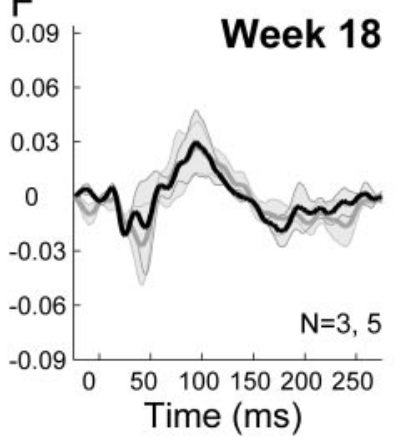

FIG. 6. Grand mean average responses to $9-\mathrm{kHz}$ tone. A: after $1 \mathrm{wk}$ of differential housing, auditory-evoked potentials from the early enriched group (experiment $2 a$ ) were indistinguishable from the late enriched group (experiment $2 b$ ). $B$ : after 2 wk of differential housing, responses of rats in the enriched (solid) environment were larger than responses from rats in the standard (gray) environment. $C$ : by 5 wk of differential housing, responses of enriched rats were 3 times the amplitude of standard housed rats. $D$ : responses were diminished within 1 wk after moving the early enriched group to the standard housing condition. $E$ : after moving to the enriched environment, the late enriched group (gray) responses were twice the amplitude of the early enriched rats (solid). $F$ : tone responses were indistinguishable when both groups were housed in the standard environment during week 18. Gray shaded regions represent the SE for each group. 


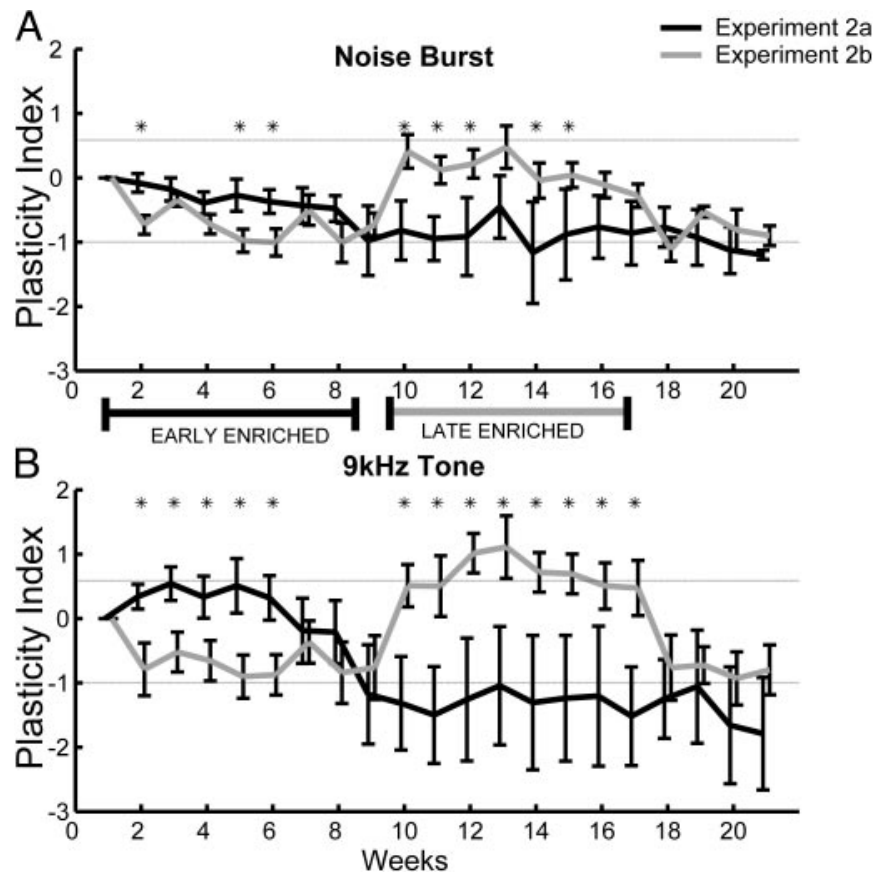

FIG. 7. Time course of environmental plasticity. Individual responses were normalized to the response recorded on week 1. Plasticity index represents the logarithm base 2 of this ratio, such that a value of 1 indicates a 2-fold increase in response strength. $A$ : noise-evoked response was up to 2-fold larger in enriched vs. standard housed rats. $B$ : tone-evoked response was up to 3 -fold larger in enriched vs. standard housed rats. *Significant differences between early and late enrichment groups. Horizontal lines indicate increases and decreases of $50 \%$ compared with week 1 . Periods for environmental enrichment for each group are indicated. The early enriched group (solid) was in the enriched environment for $8 \mathrm{wk}$, and the late enriched group (gray) was in the enriched environment beginning at week 10 of the experiment. Both groups were $4 \mathrm{wk}$ old at the beginning of the experiment and were housed in the standard environment during experimental weeks 9 and 19-21. Error bars represent $\mathrm{SE}$.

of 1 indicates a twofold increase in the magnitude of the evoked potential compared with week 1 , while a value of -1 indicates that the magnitude of the evoked potential was halved (see METHODS). While the evoked response to a noise burst was larger, enrichment caused a proportionally larger increase of tone-evoked responses. This finding indicates that stimuli selected to evoke the maximal response may not be optimal for documenting cortical plasticity.

While the average noise-evoked plasticity index for enriched and deprived rats was significantly different $(P<0.05)$, the difference was only 0.57 during weeks $2-8$ and 0.83 during weeks 10-18 (Fig. 7A). The differences in the tone-evoked plasticity indices for enriched and deprived rats were larger: 1.21 during weeks 2-8 and 1.63 during weeks 10-18 (Fig. $7 B$ ). The larger influence of environment on tone-evoked responses may be due to a ceiling effect caused by the near maximal activation of auditory neurons by noise bursts. To confirm this observation, we also recorded responses from a quiet $(50 \mathrm{~dB})$ noise burst. The scale of environmental plasticity documented using quiet noise bursts was intermediate to the plasticity observed using tones or loud noise bursts. (0.96 during weeks 2-8 and 1.49 during weeks 10-18).

Although the physiological effects of environmental enrichment were large (two- to threefold enhancement relative to standard housing), they typically did not last longer than $1 \mathrm{wk}$ after a return to the standard environment. In every case, the mean plasticity index significantly decreased when rats were moved from the enriched to the standard condition and significantly increased when rats were moved from the standard to the enriched condition (Fig. 8). There were no significant differences in the scale of plasticity that occurred in adult and young rats (Fig. 8).

\section{I S C U S S I O N}

This study was designed to evaluate the neurophysiologic consequences of environmental enrichment on auditory cortex neurons. Microelectrode mapping provides the greatest spatial precision in documenting cortical plasticity. This technique made it possible to document improvements in A1 response strength, latency, threshold, and frequency selectivity; however, neural responses from each rat could only be sampled at one time point. Evoked potentials were also recorded from auditory cortex in awake rats to document the time course of changes in response strength. Both methods confirmed that enrichment substantially enhances cortical responses. This environmental plasticity developed in $<2$ wk of enrichment and reversed after $1 \mathrm{wk}$ in standard housing. These results indicate that auditory cortex neurons in young and adult rats are remarkably sensitive to environmental conditions.

While physiological plasticity studies in humans and animals often probe neural responses with intense stimuli, more reliable plasticity may be observed in many situations using stimuli that do not elicit saturated responses (Wible et al. 2002). In this study, the greatest proportional changes in evoked response were recorded using sounds that stimulate less than the maximal neural response. A $9-\mathrm{kHz}$ tone generates an auditory evoked potential that is one-third the amplitude of a noise burst of the same intensity, because epidural recordings are influenced by neurons with a wide range of frequency preferences. Despite the better signal to noise ratio in recordings evoked by noise bursts, environmental plasticity was more reliable and proportionally larger when assayed using tones. Thus it appears that stimuli that evoke smaller neural responses can avoid a potential ceiling effect and reveal a greater influence of environment on cortical responsiveness. The environmental plasticity documented using a quiet noise burst was intermediate between the effects documented with tone and noise.

\section{Potential mechanisms}

Changes in cellular, synaptic, or network properties may contribute to the experience-dependent modifications induced by environmental conditions (Gilbert 1998; Katz and Shatz 1996; Syka 2002). Acute sampling from A1 neurons in enriched rats revealed that increased response strength was accompanied by decreased response threshold and receptive field size. Smaller receptive fields were also observed in visual and somatosensory cortex after enrichment (Beaulieu and Cynader 1990; Coq and Xerri 1998). The persistence of these effects under general anesthesia is consistent with the earlier conclusions that structural changes contribute to environmental plasticity. Reduced inhibition could explain some of the observed effects documented in this study, such as increased excitability and decreased 


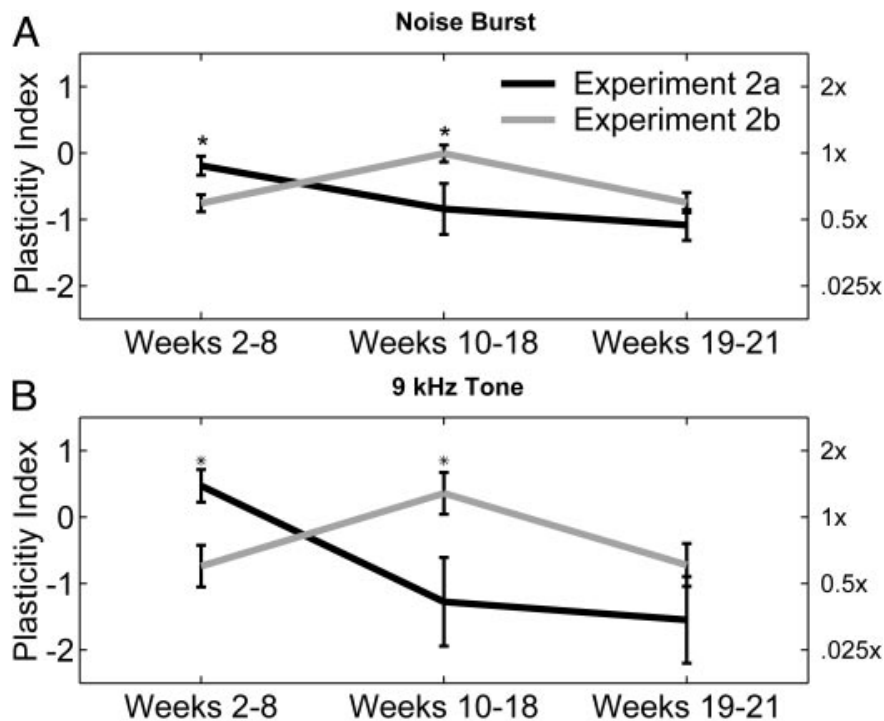

FIG. 8. Housing condition significantly affects the strength of tone and noise-evoked potentials. Mean responses evoked by both noise $(A)$ and tone $(B)$ bursts were significantly stronger in the enriched environment compared with the standard housing condition. These enhanced responses were eliminated when rats were returned to standard conditions. Environmental plasticity was equally strong in sexually immature $(<13 \mathrm{wk}$ old) and sexually mature $(>13$ wk old $)$ rats.

threshold of cortical neurons (Beaulieu and Colonnier 1987; Dykes et al. 1984; Eysel et al. 1998; Wang et al. 2000, 2002). While it appears that decreased inhibition influences physiologic properties in enriched cortex, additional mechanisms are likely to contribute to environmental plasticity.

Differences in other modulatory neurotransmitter levels and enzymes may also affect response properties in enriched cortex. Enrichment increases levels of cortical norepinephrine, dopamine, acetylcholine, and acetylcholinesterase (Feenstra et al. 1995; Giovannini et al. 2001; Naka et al. 2002; Park et al. 1992). Norepinephrine decreases receptive field size in auditory cortex neurons and acetylcholine decreases thresholds (Manunta and Edeline 1997; Metherate et al. 1990). These results indicate that changes in multiple neurotransmitter systems could explain many of our physiological findings.

Our findings that environmental plasticity develops over many days and is maintained under general anesthesia support earlier evidence of structural changes induced by environmental enrichment (van Praag et al. 2000). Both physiologic and anatomic changes can reverse with differential housing conditions. Enrichment of aged rats restored narrow receptive fields, large cortical maps, and increased response strength, typical of motor cortex in younger rats (Reinke and Dinse 1999). While some anatomical changes of enrichment persist (Camel et al. 1986), others reverse after rats are returned to standard conditions. When 1 mo of impoverished housing followed enrichment, the increase in cortical thickness reversed, but the increase in the oligodendrocyte to neuron ratio was maintained (Katz and Davies 1984). Our finding that the amplitude of the auditory evoked response is decreased within $1 \mathrm{wk}$ of return to the standard environment indicates the need for additional studies to determine whether anatomical changes associated with enrichment reverse with a similar time course.

\section{Remaining questions}

Both epidural-evoked potentials and microelectrode recordings show significant cortical plasticity. However, the proportional changes recorded with these techniques were markedly different. In awake rats, enrichment resulted in a greater than twofold increase in the tone-evoked response, while in anesthetized rats, the number of action potentials increased by only one-third. Three factors could be responsible for the greater enhancement of awake evoked potentials compared with anesthetized action potentials. First, anesthesia may eliminate possible neuromodulatory differences in awake standard and enriched rats. Second, evoked potentials may exhibit a greater capacity to increase because they are generated by summed synaptic potentials, while the action potentials recorded in the acute experiments are generated by a thresholding nonlinearity and are thus more easily saturated. Finally, it is possible that nonprimary auditory regions, which likely contribute to the evoked potentials, are more sensitive to environmental conditions than A1 (Eggermont and Ponton 2002). The latency of the N1a peak ( $\sim 25 \mathrm{~ms})$ is similar to the peak response of A1 neurons recorded with microelectrodes. The greatest changes in the evoked potentials occurred considerably later (N1b at $\sim 45 \mathrm{~ms}$ and the P1 at $\sim 75 \mathrm{~ms}$ ). This difference suggests that plasticity in auditory fields surrounding A1 may have had a substantial contribution to evoked potential plasticity. Future studies using chronically implanted microelectrode arrays to record from A1 and other auditory fields are needed to distinguish between these potential explanations.

Previous studies have shown that multiple factors influence the degree of plasticity generated by environmental enrichment. These include physical activity, enrichment duration, social experience, behavioral relevance of sensory events, and age. For example, simple wheel running has been shown to increase cell proliferation and neurogenesis in the adult mouse dentate gyrus (van Praag et al. 1999). Even a few hours of daily enrichment increased brain weight, acetylcholinesterase staining, and RNA/DNA ratios (Ferchmin and Bennett 1975; Will et al. 1977). While passive sensory enrichment failed to alter brain weight in rats, social interactions significantly increased brain weight (Ferchmin and Bennett 1975). Studies in primates have shown that focused attention is required if sensory inputs are to stimulate cortical plasticity (Ahissar and Ahissar 1994; Recanzone et al. 1993). These results indicate that both social interactions and attention contribute to the expression of cortical plasticity. The enriched environment used in this study was designed to expose rats to a wide variety of behaviorally meaningful sensory inputs. While background sounds were present in both environments, in the standard environment, these sounds had little behavioral relevance and were less diverse than the sounds in the enriched environment. The enriched housing condition was also designed to increase the behavioral relevance by providing greater social interactions than the standard environment. However, this study cannot determine whether social experience, behavioral relevance of sensory events, attention, physical activity, or enrichment duration were important factors in altering cortical responses.

Exposure to behaviorally relevant sounds that are spectrally restricted (i.e., tones) can alter A1 topography, receptive field size, and latency (Recanzone et al. 1993; Weinberger and Bakin 1998). These changes are typically restricted to the 
region of the cortical map activated by these sounds. Although some tonal stimuli were part of the environmental enrichment, the plasticity effects documented in this study were not frequency-specific and were found across A1. Sensory sensitization due to random foot shock also strengthens responses across A1 (Bakin and Weinberger 1990). However, these changes develop and fade much more quickly than the effects observed in this study and would likely not persist under the general anesthesia used in some of our experiments. Despite these differences, it remains likely that many of the same mechanisms involved in fear conditioning and perceptual learning are also involved in environmental plasticity.

Numerous studies have shown that the development of primary sensory cortex can be disrupted by abnormal sensory input in very young animals (Rema et al. 2003; Weliky and Katz 1997; Zhang et al. 2002). For example, rats exposed to pulsed noise before $4 \mathrm{wk}$ of age exhibited disruption of A1 tonotopy and decreased frequency selectivity (Zhang et al. 2002), while pulsed noise exposure after $4 \mathrm{wk}$ of age resulted in no significant plasticity. Our results indicate that cortical responses can be degraded or enhanced in animals well beyond these early sensitive periods. These results are consistent with earlier observations that highly focused behavioral training can improve cortical responses in adults. Since this study was not designed to determine how specific factors influence environmental plasticity, additional studies will be needed to evaluate the potentially interacting factors responsible for the profound physiological changes observed in this study.

The results of this and earlier studies indicate that rich and stimulating environments can significantly improve the sensory information processing of cortical neurons. Although the exact consequences of plasticity on cortical development or recovery from injury are not clear, numerous studies suggest that environmental enrichment may be useful in promoting recovery from neurological disability (Baranek 2002; Biernaskie and Corbett 2001; Jones and Schallert 1994; Klinke et al. 1999; Merzenich et al. 1996; Nudo and Friel 1999; Risedal et al. 2002).

\section{A C K N OW LE D G M EN TS}

The authors thank W. W. Dai for assistance with recording sessions and animal colony management.

\section{G R A N T S}

This work was supported by the Cure Autism Now Foundation and the Callier Excellence in Education Fund.

\section{REFERENCES}

Ahissar E and Ahissar M. Plasticity in auditory cortical circuitry. Curr Opin Neurobiol 4: 580-587, 1994.

Bakin J and Weinberger N. Classical conditioning induces CS-specific receptive field plasticity in the auditory cortex of the guinea pig. Brain Res 536: 271-286, 1990.

Baranek GT. Efficacy of sensory and motor interventions for children with autism. J Autism Dev Disord 32: 397-422, 2002.

Beaulieu C and Colonnier M. Effect of the richness of the environment on the cat visual cortex. J Comp Neurol 266: 478-494, 1987.

Beaulieu C and Cynader M. Effect of the richness of the environment on neurons in cat visual cortex. I. Receptive field properties. Brain Res Dev Brain Res 53: 71-81, 1990.

Bennett EL, Diamond MC, Morimoto H, and Hebert M. Acetylcholinesterase activity and weight measures in fifteen brain areas from six lines of rats. J Neurochem 13: 563-572, 1966.
Biernaskie $\mathbf{J}$ and Corbett $\mathbf{D}$. Enriched rehabilitative training promotes improved forelimb motor function and enhanced dendritic growth after focal ischemic injury. $J$ Neurosci 21: 5272-5280, 2001.

Camel JE, Withers GS, and Greenough WT. Persistence of visual cortex dendritic alterations induced by postweaning exposure to a "superenriched" environment in rats. Behav Neurosci 100: 810-813, 1986.

Coq JO and Xerri C. Environmental enrichment alters organizational features of the forepaw representation in the primary somatosensory cortex of adult rats. Exp Brain Res 121: 191-204, 1998.

Diamond MC. Response of the brain to enrichment. An Acad Bras Cienc 73: 211-220, 2001.

Diamond MC, Law F, Rhodes H, Lindner B, Rosenzweig MR, Krech D, and Bennett EL. Increases in cortical depth and glia numbers in rats subjected to enriched environment. J Comp Neurol 128: 117-126, 1966.

Diamond MC, Rosenzweig MR, Bennett EL, Lindner B, and Lyon L. Effects of environmental enrichment and impoverishment on rat cerebral cortex. J Neurobiol 3: 47-64, 1972.

Doron NN, Ledoux JE, and Semple MN. Redefining the tonotopic core of rat auditory cortex: physiological evidence for a posterior field. J Comp Neurol 4: 345-360, 2002.

Dykes RW, Landry P, Metherate R, and Hicks TP. Functional role of GABA in cat primary somatosensory cortex: shaping receptive fields of cortical neurons. J Neurophysiol 52: 1066-1093, 1984.

Edeline JM. Learning-induced physiological plasticity in the thalamo-cortical sensory systems: a critical evaluation of receptive field plasticity, map changes and their potential mechanisms. Prog Neurobiol 57: 165-224, 1999.

Eggermont JJ and Ponton CW. The neurophysiology of auditory perception: from single units to evoked potentials. Audiol Neurootol 7: 71-99, 2002.

Eysel UT, Shevelev IA, Lazareva NA, and Sharaev GA. Orientation tuning and receptive field structure in cat striate neurons during local blockade of intracortical inhibition. Neuroscience 84: 25-36, 1998.

Feenstra MG, Botterblom MH, and van Uum JF. Novelty-induced increase in dopamine release in the rat prefrontal cortex in vivo: inhibition by diazepam. Neurosci Lett 189: 81-84, 1995.

Ferchmin PA and Bennett EL. Direct contact with enriched environment is required to alter cerebral weights in rats. J Comp Physiol Psychol 88: 360-367, 1975.

Gilbert CD. Adult cortical dynamics. Physiol Rev 78: 467-485, 1998.

Giovannini MG, Rakovska A, Benton RS, Pazzagli M, Bianchi L, and Pepeu G. Effects of novelty and habituation on acetylcholine, GABA, and glutamate release from the frontal cortex and hippocampus of freely moving rats. Neuroscience 106: 43-53, 2001.

Globus A, Rosenzweig MR, Bennett EL, and Diamond MC. Effects of differential experience on dendritic spine counts in rat cerebral cortex. J Comp Physiol Psychol 82: 175-181, 1973.

Godde B, Berkefeld T, David-Jurgens M, and Dinse HR. Age-related changes in primary somatosensory cortex of rats: evidence for parallel degenerative and plastic-adaptive processes. Neurosci Biobehav Rev 26: 743-752, 2002.

Greenough WT, Volkmar FR, and Juraska JM. Effects of rearing complexity on dendritic branching in frontolateral and temporal cortex of the rat. Exp Neurol 41: 371-378, 1973.

Hilbig H, Bidmon HJ, Steingruber S, Reinke H, and Dinse HR. Enriched environmental conditions reverse age-dependent gliosis and losses of neurofilaments and extracellular matrix components but do not alter lipofuscin accumulation in the hindlimb area of the aging rat brain. J Chem Neuroanat 23: 199-209, 2002.

Jones TA and Schallert T. Use-dependent growth of pyramidal neurons after neocortical damage. J Neurosci 14: 2140-2152, 1994.

Katz HB and Davies CA. Effects of differential environments on the cerebral anatomy of rats as a function of previous and subsequent housing conditions. Exp Neurol 83: 274-287, 1984.

Katz LC and Shatz CJ. Synaptic activity and the construction of cortical circuits. Science 274: 1133-1138, 1996.

Kilgard MP and Merzenich MM. Cortical map reorganization enabled by nucleus basalis activity. Science 279: 1653-1654, 1998.

Kilgard MP and Merzenich MM. Distributed representation of spectral and temporal information in rat primary auditory cortex. Hear Res 134: 16-28, 1999.

Kilgard MP, Pandya PK, Vazquez JL, Gehi A, Schreiner CE, and Merzenich MM. Sensory input directs spatial and temporal plasticity in primary auditory cortex. J Neurophysiol 86: 339-353, 2001. 
Klinke R, Kral A, Heid S, Tillein J, and Hartmann R. Recruitment of the auditory cortex in congenitally deaf cats by long-term cochlear electrostimulation. Science 285: 1729-1733, 1999.

Manunta Y and Edeline JM. Effects of noradrenaline on frequency tuning of rat auditory cortex neurons. Eur J Neurosci 9: 833-847, 1997.

Merzenich M, Wright B, Jenkins W, Xerri C, Byl N, Miller S, and Tallal P. Cortical plasticity underlying perceptual, motor, and cognitive skill development: implications for neurorehabilitation. Cold Spring Harb Symp Quant Biol 61: 1-8, 1996.

Metherate R, Ashe JH, and Weinberger NM. Acetylcholine modifies neuronal acoustic rate-level functions in guinea pig auditory cortex by an action at muscarinic receptors. Synapse 6: 364-368, 1990.

Naka F, Shiga T, Yaguchi M, and Okado N. An enriched environment increases noradrenaline concentration in the mouse brain. Brain Res 924: 124-126, 2002.

Nudo RJ and Friel KM. Cortical plasticity after stroke: implications for rehabilitation. Rev Neurol 155: 713-717, 1999.

Park GA, Pappas BA, Murtha SM, and Ally A. Enriched environment primes forebrain choline acetyltransferase activity to respond to learning experience. Neurosci Lett 143: 259-262, 1992.

Rampon C, Jiang CH, Dong H, Tang YP, Lockhart DJ, Schultz PG, Tsien JZ, and Hu Y. Effects of environmental enrichment on gene expression in the brain. Proc Natl Acad Sci USA 97: 12880-12884, 2000.

Recanzone GH, Schreiner CE, and Merzenich MM. Plasticity in the frequency representation of primary auditory cortex following discrimination training in adult owl monkeys. J Neurosci 13: 87-103, 1993.

Reinke $\mathrm{H}$ and Dinse HR. Plasticity in the somatosensory and motor cortex of rats: impact of age and housing conditions. In: From Molecular Neurobiology to Clinical Neuroscience, edited by Elsner N and Eysel U. Stuttgart: Thieme, 1999, p. 409-415.

Rema V, Armstrong-James M, and Ebner FF. Experience-dependent plasticity is impaired in adult rat barrel cortex after whiskers are unused in early postnatal life. J Neurosci 23: 358-366, 2003.

Risedal A, Mattsson B, Dahlqvist P, Nordborg C, Olsson T, and Johansson BB. Environmental influences on functional outcome after a cortical infarct in the rat. Brain Res Bull 58: 315-321, 2002.

Schapiro S and Vukovich KR. Early experience effects upon cortical dendrites: a proposed model for development. Science 167: 292-294, 1970.
Sirevaag AM and Greenough WT. Differential rearing effects on rat visual cortex synapses. III. Neuronal and glial nuclei, boutons, dendrites, and capillaries. Brain Res 424: 320-332, 1987.

Staiger JF, Masanneck C, Bisler S, Schleicher A, Zuschratter W, and Zilles K. Excitatory and inhibitory neurons express c-Fos in barrel-related columns after exploration of a novel environment. Neuroscience 109: 687699, 2002.

Syka J. Plastic changes in the central auditory system after hearing loss, restoration of function, and during learning. Physiol Rev 82: 601-636, 2002.

van Praag H, Kempermann G, and Gage FH. Running increases cell proliferation and neurogenesis in the adult mouse dentate gyrus. Nat $\mathrm{Neu}$ rosci 2: 266-270, 1999.

van Praag H, Kempermann G, and Gage FH. Neural consequences of environmental enrichment. Nat Rev Neurosci 1: 191-198, 2000.

Volkmar FR and Greenough WT. Rearing complexity affects branching of dendrites in the visual cortex of the rat. Science 176: 1145-1147, 1972.

Wang J, Caspary D, and Salvi RJ. GABA-A antagonist causes dramatic expansion of tuning in primary auditory cortex. Neuroreport 11: 1137-1140, 2000.

Wang J, McFadden SL, Caspary D, and Salvi R. Gamma-aminobutyric acid circuits shape response properties of auditory cortex neurons. Brain Res 944 219-231, 2002.

Weinberger NM and Bakin JS. Learning-induced physiological memory in adult primary auditory cortex: receptive fields plasticity, model, and mechanisms. Audiol Neurootol 3: 145-167, 1998.

Weliky M and Katz LC. Disruption of orientation tuning in visual cortex by artificially correlated neuronal activity. Nature 386: 680-685, 1997.

Wible B, Nicol T, and Kraus N. Abnormal neural encoding of repeated speech stimuli in noise in children with learning problems. Clin Neurophysiol 113: 485-494, 2002.

Will BE, Rosenzweig MR, Bennett EL, Hebert M, and Morimoto $H$. Relatively brief environmental enrichment aids recovery of learning capacity and alters brain measures after postweaning brain lesions in rats. J Comp Physiol Psychol 91: 33-50, 1977.

Zhang LI, Bao S, and Merzenich MM. Disruption of primary auditory cortex by synchronous auditory inputs during a critical period. Proc Natl Acad Sci USA 99: 2309-2314, 2002. 